\title{
Solar modulation during the Holocene
}

\author{
F. Steinhilber, J. A. Abreu, and J. Beer \\ Eawag, Aquatic Science, Surface Waters - Radioactive Tracers, Überlandstrasse 133, 8600 Dübendorf, Switzerland
}

Received: 22 August 2007 - Revised: 22 November 2007 - Accepted: 22 November 2007 - Published: 5 February 2008

\begin{abstract}
We built a composite of three reconstructions of the solar modulation function over the Holocene. The reconstructions until 1950 are based on data from cosmogenic radionuclides and the present time (1951-2004) on neutron monitor data.

Interpreting our composite as an index of solar activity, we were able to compare the current solar activity with the last 9300 years. During this time span 25 periods with similar high activity than the current period were found. That corresponds to about $15 \%$ of the time which lead to the conclusion that currently the Sun is very but not exceptionally active.

Our composite has a large potential for studies dealing with solar activity like the understanding of the solar dynamo and the reconstruction of solar forcing.
\end{abstract}

\section{Introduction}

Direct continuous observations of the solar activity have been started in about 1610 with the invention of the telescope. About 200 years later the famous Schwabe cycle was discovered. Additionally a long-term trend could be seen in the sunspot record. Soon people noticed a similarity between this long-term trend and temperature records. In particular periods which are characterized by a lack of sunspots such as the Maunder minimum (1645-1715) seem to coincide with cold climate conditions. To study this correlation a large variety of time series of solar parameters have been continuously recorded in more recent times.

Unfortunately those time series do not provide any information about long-term trends. Hence they can not answer the question whether the Maunder minimum was a very exceptional state or if such periods have been quite frequent, nor if the current solar maximum is very special or quite

Correspondence to: F. Steinhilber

(friedhelm.steinhilber@eawag.ch) common. However this information is very important for improving the solar dynamo models and estimating the past solar forcing. For this answer accurate solar activity reconstructions going as far back in time as possible are needed. So far only cosmogenic radionuclides can provide this information on millennial time scales. Such reconstructions were made several times.

Solanki et al. (2004) reconstruct sunspots for the last 11000 years based on the cosmogenic radionuclide ${ }^{14} \mathrm{C}$ from tree rings. Their main result is that the current solar activity is exceptionally high. Their study shows that the last period when the Sun was as active as today was 8000 years ago and that in $10 \%$ of the time the Sun was similar active as today.

Vonmoos et al. (2006) compare the solar modulation functions $\Phi$ during the Holocene (period $340-9300 \mathrm{BP}$ ) using ${ }^{10} \mathrm{Be}$ from the Greenland Ice core Project (GRIP) ice core with ${ }^{14} \mathrm{C}$ from tree rings. They find slightly different $\Phi$ values for the two radionuclides which points to different non-solar signals in these proxies. But nevertheless both $\Phi$ records show similar long-term trends. Unfortunately a comparison of the Sun during the Holocene with the current time is impossible since their reconstruction does not reach to today.

Muscheler et al. (2007) use the radionuclides ${ }^{10} \mathrm{Be}$ and ${ }^{14} \mathrm{C}$ to reconstruct the solar modulation function $\Phi$ for the past 1000 years and come to a somewhat different result than Solanki et al. (2004). Their conclusion is that compared to the last 1000 years the recent solar activity is high but not exceptionally high.

In order to resolve the discrepancies between the different long-term trends in the reconstructions we constructed a composite of reconstructions of the solar modulation function bridging the values of Vonmoos et al. (2006) (cosmogenic radionuclide ${ }^{10} \mathrm{Be}$ ) with the modern instrumental data (neutron monitor data). 


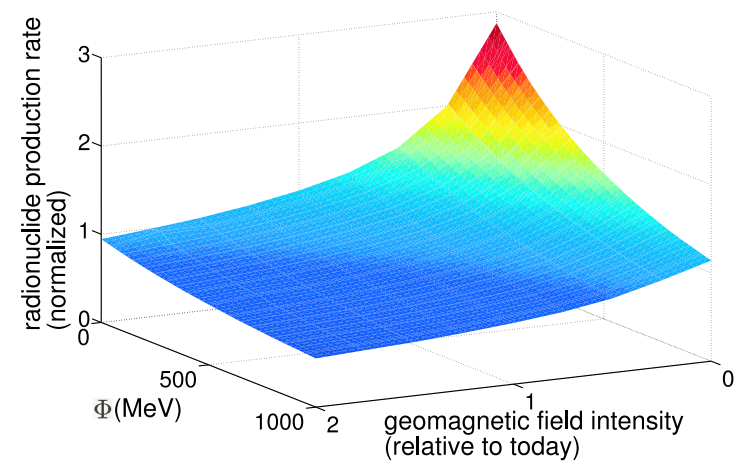

Fig. 1. Radionuclide production rate as a function of geomagnetic field intensity and solar modulation function $\Phi$. In the calculation, described in detail in Masarik and Beer (1999), protons and $\alpha$-paticles are considered.

\section{Theory: Solar modulation}

The production of cosmogenic radionuclides depends on the intensity of cosmic ray particles (CRs) penetrating into the Earth's atmosphere. Before CRs reach the Earth, they have to pass the heliosphere in which they are modulated.

The modulation of CRs is composed of different processes such as diffusion, adiabatic cooling, convection and drifts. These processes are included in the CR transport equation formulated by Parker (1965) which describes the propagation of CRs through the heliosphere and allows for calculating the CR spectrum everywhere within the heliosphere.

Gleeson and Axford (1968) found an approximation of that equation the so-called force-field approximation

$J(T, \Phi)=J_{\mathrm{LIS}}(T+\Phi) \frac{T\left(T+2 T_{0}\right)}{(T+\Phi)\left(T+\Phi+2 T_{0}\right)}$

where $J_{\text {LIS }}$ is the local interstellar CR spectrum, $T$ the kinetic energy of the CRs and $T_{0}$ their rest energy. $\Phi$ describes the modification of the local interstellar spectrum (LIS) in the heliosphere. It has the unit of energy and is called solar modulation function.

As can be easily seen from Eq. (1) for $\Phi=0$ the modulation effect vanishes. The higher the solar modulation the less CRs reach the Earth's atmosphere and the less cosmogenic radionuclides (like ${ }^{10} \mathrm{Be}$ ) are produced.

The usefulness of $\Phi$ to describe the CR spectra at $1 \mathrm{AU}$ is shown by Caballero-Lopez and Moraal (2004). They compare experimental CR data with the spectrum obtained from Eq. (1) and with the spectrum calculated with the CR transport equation. They come to the conclusion that $\Phi$ is a good parameter to describe solar modulation at Earth (1 AU), if the considered CR particles have energies above $0.1 \mathrm{GeV} /$ nucleon. This condition is fulfilled for the production of ${ }^{10} \mathrm{Be}$ as shown by McCracken (2004b).

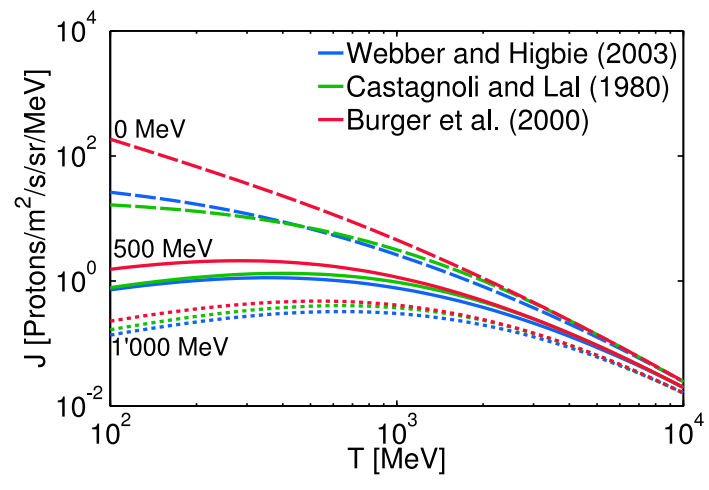

Fig. 2. CR spectra calculated with Eq. (1) using the local interstellar spectra of Webber and Higbie (2003), Castagnoli and Lal (1980) and Burger et al. (2000) for different values of the solar modulation function $\Phi=0$ (dashed), $500 \mathrm{MeV}$ (solid line) and $1000 \mathrm{MeV}$ (dotted). The cosmic ray energies $T$ shown here are responsible for most of the ${ }^{10} \mathrm{Be}$ production (McCracken, 2004b).

\section{Theory: Cosmogenic radionuclides ${ }^{10} \mathrm{Be}$ and solar modulation}

The radionuclide signal from ice cores is composed of three components: (1) solar activity (described by $\Phi)$, (2) geomagnetic field intensity (mainly dipole moment component) and (3) system effects (geochemical behaviour).

Since we focus here on the solar activity the other two components have to be identified and removed.

The system component is the result of the transport of the radionuclides from the atmosphere to the terrestrial archive. They depend strongly on the geochemical properties of the considered radionuclide and the prevailing climatic conditions like the precipitation rate. McCracken (2004b) analyzes different averages of ${ }^{10} \mathrm{Be}$ data from Greenland and Antarctic and shows that the system effect component contributes less than $10 \%$ to the total signal when the data is averaged over 22 or more years.

The geomagnetic field acts as a shield for the charged CRs. Since its intensity varied significantly during the Holocene (shown in the top panel of Fig. 5) it has also a significant effect on the radionuclide production and must be considered. This is done by using reconstructions of the geomagnetic field intensity. The magnetic field intensity is used to work out the so-called cutoff rigidity which says how much energy a CR needs to cross through the Earth's magnetosphere.

To conclude the production rate of cosmogenic radionuclides over the Holocene depends on the solar magnetic field described by the solar modulation function $\Phi$ and the geomagnetic field intensity. As can be seen in Fig. 1 this dependence is non-linear. Here we used the calculations by Masarik and Beer (1999) based on Monte Carlo techniques. 
Table 1. Reconstructions of the solar modulation function $\Phi$ used for the composite, the data and source (NM=neutron monitor, GRIP = Greenland Ice core Project), the LIS they are based on, covered time period, time resolution and the maximal standard deviation (SD). The SD from Vonmoos et al. (2006) and FAA (US Federal Aviation Administration) are originally given in percentage. The reconstruction by FAA described in O'Brien et al. (1996) is not used for the composite but for a comparison with the reconstruction by Usoskin et al. (2005).

\begin{tabular}{lllrrr}
\hline Reconstruction of $\Phi$ & Data & used LIS & $\begin{array}{c}\text { Period } \\
\text { years (AD) }\end{array}$ & $\begin{array}{r}\text { Resolution } \\
\text { max. SD } \\
(\mathrm{MeV})\end{array}$ \\
\hline Usoskin et al. (2005) & $\mathrm{NM} /$ multiple stations & Burger et al. (2000) & $1951-2004$ & monthly/annual & 60 \\
McCracken et al. (2004a) & ${ }^{10}$ Be/Southpole & Webber and Higbie (2003) & $850-1958$ & $\begin{array}{r}22 \text {-year averages } \\
40\end{array}$ \\
Vonmoos et al. (2006) & ${ }^{10}$ Be/GRIP & Castagnoli and Lal (1980) & $-7363-1645$ & 25 -year running mean & $80(10 \%)$ \\
\hline FAA (O'Brien et al., 1996) & NM/Deep River & Castagnoli and Lal (1980) & $1958-2007$ & monthly/annual & $60(6 \%)$ \\
\hline
\end{tabular}

\section{Data}

This work is based on three reconstructions of the solar modulation function $\Phi$ summarized in Table 1. They were chosen because together they cover the period from today back to $9300 \mathrm{BP}$.

In all reconstructions the influence of the geomagnetic field is considered. Vonmoos et al. (2006) use the reconstruction of geomagnetic dipole component by Yang et al. (2000) and McCracken (2004b) make use of the geomagnetic field by McElhinny and McFadden (2000) who use in principle the same proxy data as Yang et al. (2000). Usoskin et al. (2005) use different cutoff rigidities depending on the location of the neutron monitor. Because the geomagnetic field has not changed significantly during their reconstruction period $(1951-2004)$, the cutoff rigidities are kept constant.

The maximal standard deviation SD of the reconstruction by McCracken et al. (2004a) is given to be $40 \mathrm{MeV}$. Usoskin et al. (2005) give values for the maximal SD which are $40 \mathrm{MeV}$ after 1973 and $60 \mathrm{MeV}$ for the period 1951 to 1973. The maximal SD of Vonmoos et al. (2006) is $80 \mathrm{MeV}$ which takes into account the uncertainties of the ${ }^{10} \mathrm{Be}$ AMS (accelerator mass spectrometry) measurement and the geomagnetic field intensity reconstruction.

Unfortunately the three reconstructions are based on different local interstellar spectra (LIS) what leads to slightly different $\Phi$ values for the same radionuclide production rate. Hence we have to correct for the different LIS before we can build the composite.

\section{Method of LIS-correction}

To solve Eq. (1) the CR spectrum at $1 \mathrm{AU}$ and the LIS have to be known. The CR spectrum can be worked out from cosmogenic radionuclide production rate data $\left({ }^{10} \mathrm{Be}\right.$ concentrations, neutron monitor count rates), but the LIS, which describes the unmodulated $\mathrm{CR}$ spectrum outside the heliosphere, has to be estimated from CR intensity measurements inside the heliosphere. Different LIS are proposed to describe those measurements. The LIS estimates differ depending on the energy as shown in Fig. 2.

Usoskin et al. (2005) give correction equations to convert $\Phi$ calculated with one LIS into $\Phi$ using another LIS. Their correction equations are obtained by comparing $\Phi$ derived from neutron monitor data. Here we used a more theoretical approach which is based only on Eq. (1) and artificial values of $\Phi$. Artificial means that we did not use experimental data but that we varied $\Phi$ in the interval of experimental data.

We did the following steps to obtain the LIS-correction equations:

1. Calculate the CR spectrum $J_{0}$ for a reference $\mathrm{LIS}=\mathrm{LIS}_{0}$ with a fixed $\Phi_{0}$ with Eq. (1)

2. Calculate the CR spectrum $J_{x}$ for a $\operatorname{LIS}_{x}$ with a varying $\Phi_{x}$ with Eq. (1) and find the CR spectrum $J_{x}$, fit which fits best the spectrum obtained with the reference LIS $_{0}$ calculated in step 1 .

3. Save $\Phi_{x}\left(J_{x, \text { fit }}\right)$ as a function of $\Phi_{0}$

4. Repeat steps 1 to 3 for different $\Phi_{0}$

5. Obtain the LIS-correction equation by applying a linear fit: $\Phi_{x}\left(J_{x, \text { fit }}\right)=m \cdot \Phi_{0}\left(J_{0}\right)+b$

As reference LIS $_{0}$ we chose the LIS by Castagnoli and Lal (1980). The index $x$ in $\operatorname{LIS}_{x}$ and $\Phi_{x}$ means: $x=\mathrm{C} 80$ LIS by Castagnoli and Lal (1980), $x=$ B00 LIS by Burger et al. (2000), $x=$ W03 LIS by Webber and Higbie (2003). $\Phi_{0}$ was varied from 0 to $1000 \mathrm{MeV}$. Corresponding to the response function of ${ }^{10} \mathrm{Be}$ given in McCracken (2004b), only protons with kinetic energies $T$ between $0.5 \mathrm{GeV} /$ nucleon and $5 \mathrm{GeV} /$ nucleon were considered.

In Fig. 3 the solar modulation functions $\Phi_{x}$ using the LIS by Burger et al. (2000) and Webber and Higbie (2003) are shown as a function of $\Phi_{0}=\Phi_{\mathrm{C} 80}$. It can be seen that the values of $\Phi_{\mathrm{B} 00}$ are higher than the $\Phi_{\mathrm{C} 80}$ values using the reference LIS. In contrast the values of $\Phi_{\mathrm{W} 03}$ are shifted to smaller values. 


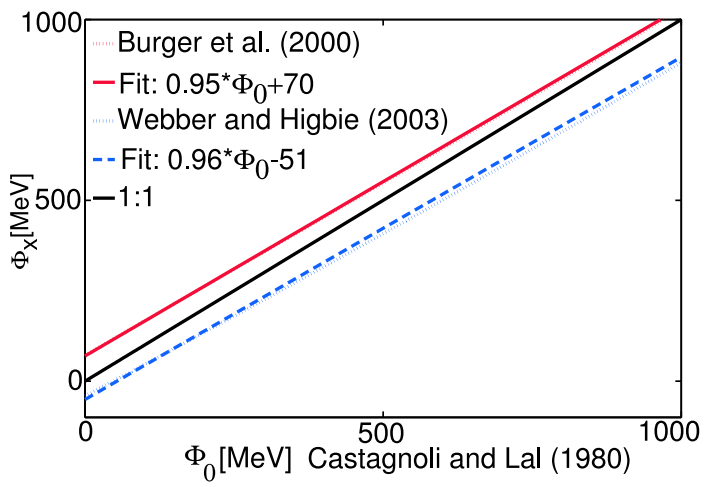

Fig. 3. Dependence of the solar modulation function $\Phi_{x}$ using the LIS by Burger et al. (2000) and Webber and Higbie (2003) on $\Phi_{0}=\Phi_{\mathrm{C} 80}$ using the reference LIS by Castagnoli and Lal (1980). In a first order approximation the solar modulation functions $\Phi$ calculated using different LIS can be converted into each other by linear functions.

As can be seen in a first order approximation the values of $\Phi$ using different LIS can be converted into each other by using linear functions which are called here LIS-correction equations. The LIS-correction equations to convert $\Phi_{x}$ to the reference $\Phi_{\mathrm{C} 80}$ are:

$$
\begin{aligned}
& \Phi_{\mathrm{C} 80}=1.04 \cdot \Phi_{\mathrm{B} 00}-73 \mathrm{MeV} \\
& \Phi_{\mathrm{C} 80}=1.05 \cdot \Phi_{\mathrm{W} 03}+54 \mathrm{MeV}
\end{aligned}
$$

By combining these equations one can also find an equation to convert $\Phi_{\mathrm{B} 00}$ into $\Phi_{\mathrm{W} 03}$. Our correction equations compare well with Usoskin et al. (2005) (their Eqs. (A4)).

Our LIS-correction Eq. (2) was tested by comparing the reconstruction of Usoskin et al. (2005) with and without using the LIS-correction with a reconstruction of $\Phi$ by FAA (US Federal Aviation Administration) also based on neutron monitor data but using our reference LIS. The calculations of the FAA are described in O'Brien et al. (1996) and the data can be found at http://www.faa.gov/education_research/ research/med_humanfacs/aeromedical/media/cari6exe.zip.

Figure 4 shows the relative differences between the data by FAA and by Usoskin et al. (2005) with and without the correction. The frequency distribution confirms the correction leading to an average relative difference of zero.

The LIS-corrections from Eqs. (2) and (3) were used to correct the reconstructions of $\Phi$ in Table 1 for the different LIS. After all $\Phi$ values were corrected to the LIS by Castagnoli and Lal (1980) they were linearly interpolated to annual resolution to obtain a homogeneous time resolution for the whole reconstruction period. Finally a running-mean of 25 years was applied. The whole composite record which covers the past 9300 years is shown in Fig. 5.

From the histogram in Fig. 6 we found the lowest value of the current solar activity to be $600 \mathrm{MeV}$. Defining that

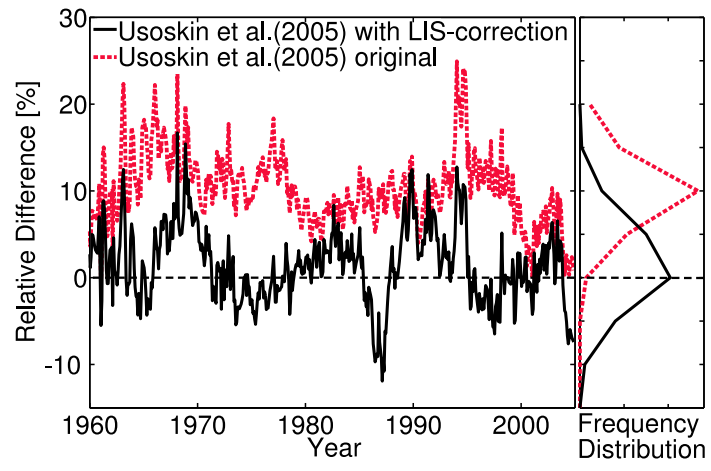

Fig. 4. Relative difference of monthly averages of the solar modulation function $\Phi$ by US Federal Aviation Administration (FAA) and by Usoskin et al. (2005) without (original) and with LIS-correction using Eq. (2). left: in time; right: frequency distribution

Table 2. Periods with $\Phi$ values above the lowest value of the 25year running mean during the period 1951-2004 $(\Phi=600 \mathrm{MeV})$, their durations and mean $\Phi$ values. Periods in bold lasted as long as the current period.

\begin{tabular}{rccc}
\hline $\begin{array}{r}\text { Period } \\
\text { no. }\end{array}$ & $\begin{array}{c}\text { Period } \\
\text { (year BP) }\end{array}$ & $\begin{array}{c}\text { Duration } \\
\text { (years) }\end{array}$ & $\begin{array}{c}\text { Mean } \Phi \\
(\mathrm{MeV})\end{array}$ \\
\hline 1 & $8794-8745$ & 49 & 695 \\
2 & $8665-8638$ & 27 & 624 \\
3 & $8433-8412$ & 21 & 619 \\
4 & $5788-5760$ & 28 & 616 \\
5 & $5506-5492$ & 14 & 617 \\
6 & $5381-5362$ & 19 & 617 \\
7 & $5114-5101$ & 13 & 607 \\
8 & $4073-4018$ & 55 & 699 \\
9 & $3943-3914$ & 29 & 625 \\
10 & $3843-3816$ & 27 & 614 \\
11 & $3554-3533$ & 21 & 613 \\
12 & $2849-2811$ & 38 & 663 \\
13 & $2590-2534$ & 56 & 678 \\
$\mathbf{1 4}$ & $\mathbf{2 4 7 1 - 2 3 8 7}$ & $\mathbf{8 4}$ & $\mathbf{7 1 2}$ \\
15 & $2277-2249$ & 28 & 623 \\
16 & $2207-2143$ & 64 & 692 \\
17 & $1992-1940$ & 52 & 647 \\
18 & $1797-1744$ & 53 & 668 \\
$\mathbf{1 9}$ & $\mathbf{1 6 8 0 - 1 5 9 9}$ & $\mathbf{8 1}$ & $\mathbf{7 9 0}$ \\
20 & $1459-1427$ & 32 & 628 \\
21 & $1132-1100$ & 32 & 622 \\
22 & $1019-981$ & 38 & 654 \\
23 & $734-700$ & 34 & 637 \\
24 & $601-562$ & 39 & 681 \\
25 & $217-206$ & 11 & 617 \\
today (26) & $\mathbf{2 5 - - 5 4}$ & $\mathbf{8 0}$ & $\mathbf{6 5 0}$ \\
\hline & & &
\end{tabular}

value as lower limit of high solar activity we found 26 periods of high solar activity in the composite. These periods are listed in Table 2. When using the given periods one must 


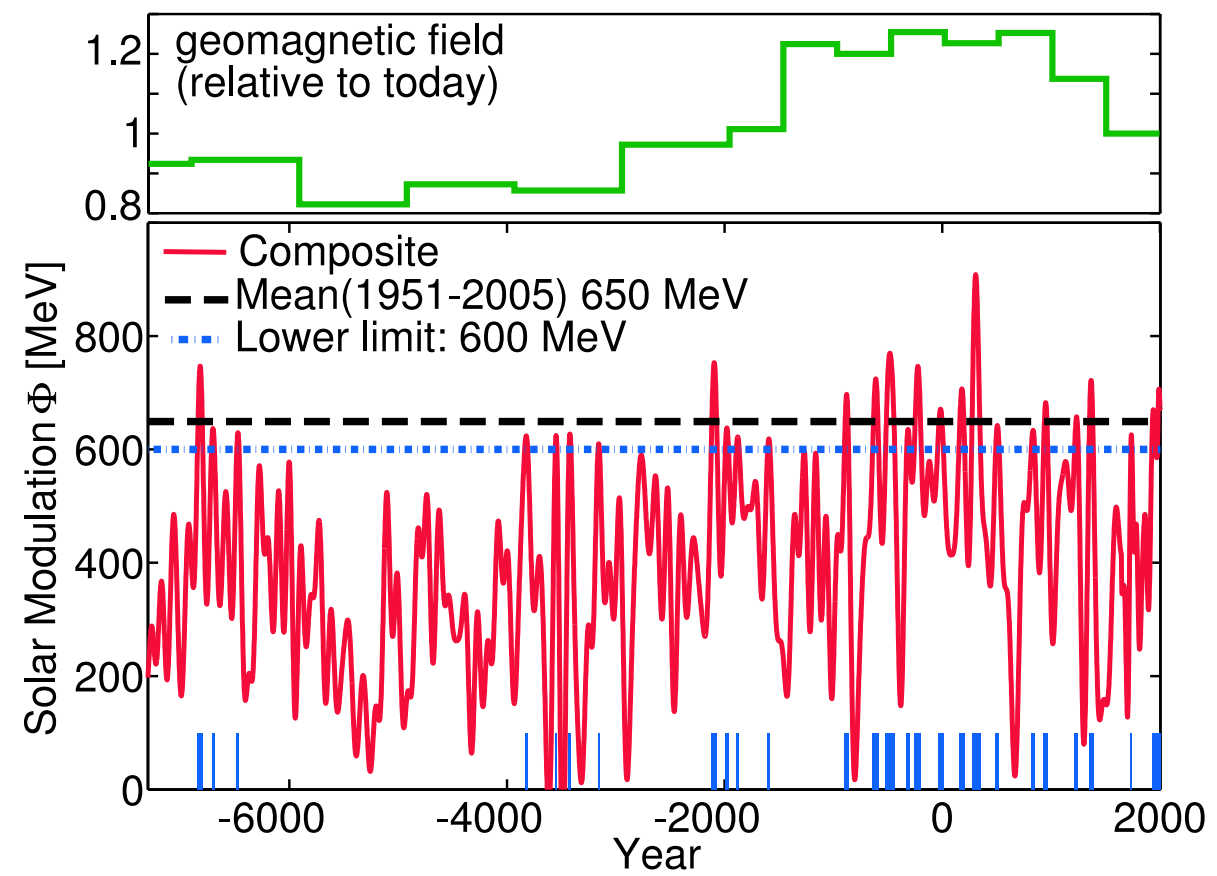

Fig. 5. Upper panel: Reconstruction of geomagnetic field intensity relative to today by Yang et al. (2000). Lower panel: Composite of 25-year running means of $\Phi$ for the Holocene (red). The black dashed line shows the mean value of the 25-year running mean $(\Phi=650 \mathrm{MeV})$ of the current Sun (1951-2004). The blue dotted dashed line is the lower limit $(\Phi=600 \mathrm{MeV})$ defining periods with high solar activity. The bars in the bottom show the periods with high solar activity $(\Phi \geq 600 \mathrm{MeV})$.

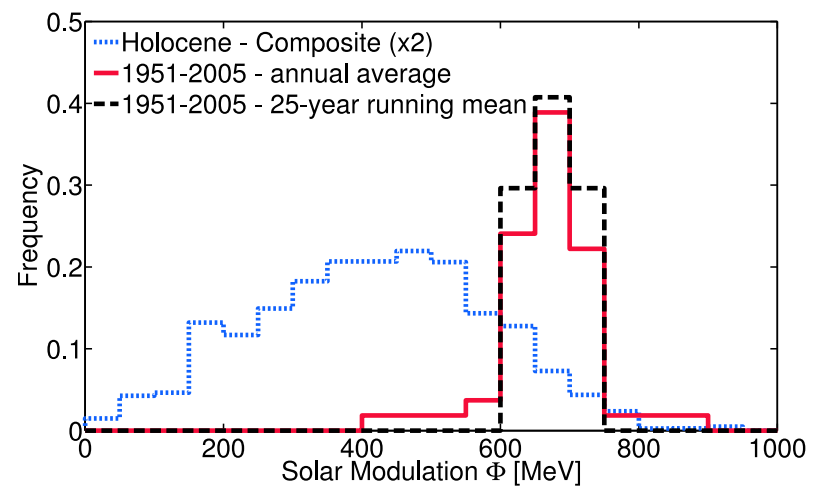

Fig. 6. Histogram of $\Phi$ values: during the Holocene 25-years running mean (blue) and for the current time 1951-2004, 1-year averages (red) and 25-year averages (black). For better visualization the frequencies of the Holocene (blue) were multiplied by a factor of two.

be aware of the absolute uncertainties ( $\leq 50$ years) of the applied timescale (GRIP timescale ss09, Johnsen et al. (1997)). However the absolute uncertainties have only a minor influence on the duration of the periods.

\section{Discussion}

Interpreting the solar modulation function as an index of solar activity allows for comparing the current solar activity with the last 9300 years.
The histogram in Fig. 6 compares the frequency distribution of $\Phi$ for the whole composite with the last 50 years. In about $85 \%$ of the time the Sun was less, in $13 \%$ equal and in about $2 \%$ more active than today. Thus we found that in $15 \%$ of the time the Sun was in a similar or even more active state as today which is a higher percentage compared to $10 \%$ found by Solanki et al. (2004).

As can be seen from Table 2 during the last 9300 years 26 periods (including today) occurred with high solar activity. Two of these periods lasted as long as the current period. The last period which showed similar high activity and also lasted as long as the current one was about 1700 years ago. We found periods (No. 23-25 in Table 2) of high activity during the last 1000 years which is in agreement with Muscheler et al. (2007).

\section{Conclusions}

Solar activity can be reconstructed using cosmogenic radionuclides from terrestrial archives provided that geomagnetic intensity variability and climate effects are removed from the data.

McCracken et al. (2004a), Usoskin et al. (2005) and Vonmoos et al. (2006) make use of cosmogenic radionuclides and reconstructed the solar modulation function for different periods. In this study a composite of these three data sets was built over most of the Holocene. Due to the fact that the three reconstructions are based on different local interstellar spectra, LIS-correction equations were derived which then were used to build the composite. 
The LIS-correction equation between the LIS by Burger et al. (2000) and Castagnoli and Lal (1980) was confirmed by a comparison of the reconstruction by Usoskin et al. (2005) with a reconstruction by the US Federal Aviation Administration.

The record provides the basis to study the solar activity in the past, to improve solar dynamo models and hopefully to develop a quantitative solar forcing function.

Acknowledgements. The authors would like to thank Horst Fichtner for his helpful comments which improved this paper. This work was financially supported by NCCR Climate - Swiss Climate Research and by the ETH Zurich poly-project: Variability of the Sun and Global Climate.

Edited by: H.-J. Fahr

Reviewed by: H. Fichtner and another anonymous referee

\section{References}

Burger, R. A., Potgieter, M. S., and B. Heber: Rigidity dependence of cosmic ray proton latitudinal gradients measured by the Ulysses spacecraft: Implications for the diffusion tensor, J. Geophys. Res., 105, A12, 27447-27456, doi: 10.1029/2000JA000153, 2000.

Caballero-Lopez, R. A. and Moraal, H.: Limitations of the force field equation to describe cosmic ray modulation, J. Geophys. Res., 109, A01101, doi:10.1029/2003JA010098, 2004.

Castagnoli, G. and Lal, D.: Solar Modulation Effects in Terrestrial Production of Carbon-14, Radiocarbon, 22, 133-158, 1980.

Gleeson, L. J. and Axford, W. L.: Solar Modulation of Galactic Cosmic Rays, Astrophys. J., 154, 1011-1026, 1968.

Johnsen, S. J., Claasen, H. B., Dansgaard, W., Gundestrup, N. S., Hammer, C. U., Andersen, U., Andersen, K. K., Hvidberg, C. S., Dahl-Jensen, D., Steffensen, J. P., Shoji, H., Sveinbjörnsdóttir, Á. E., White, J., Jouzel, J., and Fisher, D.: The $\delta^{18}$ O record along the Greenland Ice Core Project deep ice core and the problem of possible Eemian climatic instability, J. Geophys. Res., 102, 26397-26410, doi:10.1029/97JC00167, 1997.
Masarik, J., and Beer, J.: Simulation of particle fluxes and cosmogenic nuclide production in the Earth's atmosphere, J. Geophys. Res., 104, 12 099-12 111, 1999.

McCracken, K. G., McDonald, F. B., Beer, J., Raisbeck, G., and Yiou, F.: A phenomenological study of the long-term cosmic ray modulation, 850-1958 AD, J. Geophys. Res., 109, A12103, doi:10.1029/2004JA010685, 2004a.

McCracken, K. G.: Geomagnetic and atmospheric effects upon the cosmogenic ${ }^{10} \mathrm{Be}$ observed in polar ice, J. Geophys. Res., 109, A04101, doi:10.1029/2003JA010060, 2004b.

McElhinny, M. W. and McFadden, P. L.: Paleomagnetism: Continents and Drifts, Academic, San Diego, California, 2000.

Muscheler, R., Joos, F., Beer, J., Müller, S. A., Vonmoos, M., and Snowball, I.: Solar activity during the last $1000 \mathrm{yr}$ inferred from radionuclide records, Qua. Sci. Rev., 26, 82-97, doi:10.1016/j.quascirev.2006.07.012, 2007.

O'Brien, K., Friedberg, W., Sauer, H.H., and Smart, D.F.: Atmospheric cosmic rays and solar energetic particles at aircraft altitudes, Env. Inter., 22, 9-44, 1996.

Parker, E. N.: The passage of energetic charged particles through interplanetary space, Planet. Space Sci., 13, 9-49,1965.

Solanki, S. K., Usoskin, I. G., Kromer, B., Schüssler, M., and Beer, J.: Unusual activity of the Sun during recent decades compared to the previous 11000 years, Nature, 431, 1084-1087, doi:10.1038/nature02995, 2004.

Usoskin, I. G., Alanko-Huotari, K., Kovaltsov, G. A., and Mursula, K.: Heliospheric modulation of cosmic rays: Monthly reconstruction for 1951-2004, J. Geophys. Res., 110, A12108, doi:10.1029/2005JA011250, 2005.

Vonmoos, M., Beer, J., and Muscheler, R.: Large variations in Holocene solar activity: Constraints from ${ }^{10} \mathrm{Be}$ in the Greenland Ice Core Project ice core, J. Geophys. Res., 111, A10105, doi:10.1029/2005JA011500, 2006.

Webber, W. R., and Higbie, P. R.: Production of cosmogenic Be nuclei in the Earth's atmosphere by cosmic rays: Its dependence on solar modulation and the interstellar cosmic ray spectrum, J. Geophys. Res., 108, 1355-1365, doi:10.1029/2003JA009863, 2003.

Yang, S., Odah, H., and Shaw, J.: Variations in the geomagnetic dipole moment over the last 12000 years, Geophys. J. Int., 140, 158-162, 2000. 